\title{
$\mathrm{HS}$
}

\section{Food insecurity in Canada and opportunities
chronic disease prevention and management}

Jordan Mak

Department of Nutritional Sciences, University of Toronto

Food insecurity, the inadequate or insecure access to food due to financial constraints, affects $12.6 \%$ of households or approximately 4 million individuals in Canada [1]. This modifiable social determinant of health independently affects chronic disease at all stages of life. Bold action to combat food insecurity in Canada has the potential to considerably reduce the prevalence of chronic disease and curb healthcare spending. However, Canada currently has no national strategy to address this public health problem and food insecurity prevalence has changed little over the past 10 years.

Since the first Canadian survey measuring hunger, data have consistently demonstrated a relationship between food insecurity and chronic disease after adjustment for household composition and income. Canadian children who experience even a single episode of hunger are more likely to have poorer health than those who never went hungry [2]. Furthermore, youth who experience multiple episodes of hunger are at a higher risk of being diagnosed with a chronic condition. For some, the effects on their well-being persist many years after experiencing hunger, leaving permanent impacts on their health.

A similar relationship exists when observing the proportion of Canadian adults with chronic physical and mental health conditions such as diabetes, heart disease, depression and anxiety. Compared to $9 \%$ of adults in food secure households, $34 \%$ of adults in severely food insecure households report a diagnosis of 3 or more chronic illnesses (Figure 1) [3]. Higher levels of stress and inadequate nutrient intake in food insecure populations are two mechanisms which may explain these observations.

The healthcare burden for food insecure individuals in Canada's publicly-funded healthcare system is a concern for all Canadians. For example, Ontarians in severely food insecure households consume healthcare services costing more than twice that of food secure individuals, including costs for physician services, inpatient stays and emergency room visits [4]. In fact, food insecurity is the single strongest predictor for high-cost healthcare use above education, region of residence, immigration status and even income [5]. This is because food insecurity is a more direct measure of material deprivation - a combined function of assets, savings, shelter costs, food costs and income itself.

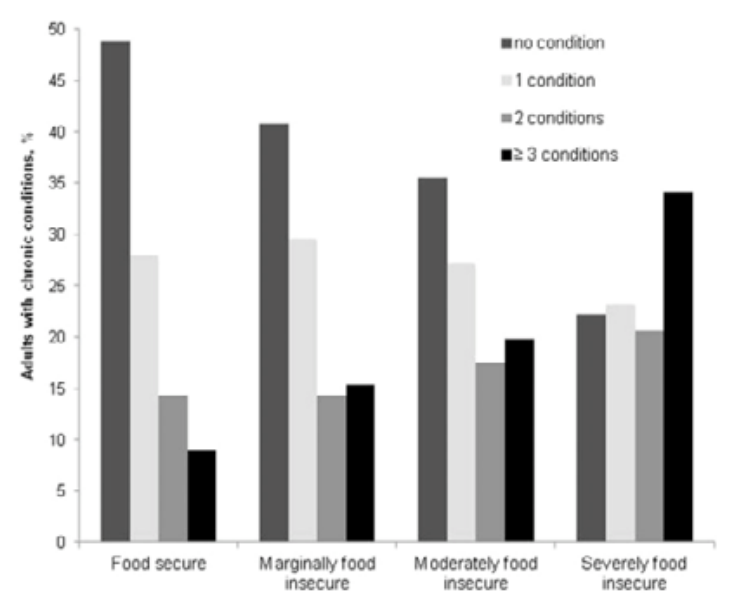

Figure 1: Prevalence of number of chronic conditions among adults, 18-64 years of age, by household food security status (Canadian Community Health Survey, 2007-2008 [3]).

Currently, there are no federal or provincial policies to explicitly reduce food insecurity. The main response by Canadians has been in the form of food charity, an ineffective initiative at addressing the root causes of hunger. Research suggests only a quarter of food insecure households use food bank services and that utilizing these services does not result in food security [6].

Food banks are an unregulated charitable model, providing food often deemed inappropriate for retail sale [7]. Users have reported misalignment between food bank provisions and household needs, inadequate amounts of food due to rationing, stigma associated with the use of charity and sporadic access due to dependence on volunteerdriven labour [7]. The lack of coordinated governmental response represents a missed opportunity to address social determinants of chronic disease in Canada.

Canadian policies to provide support to the most 


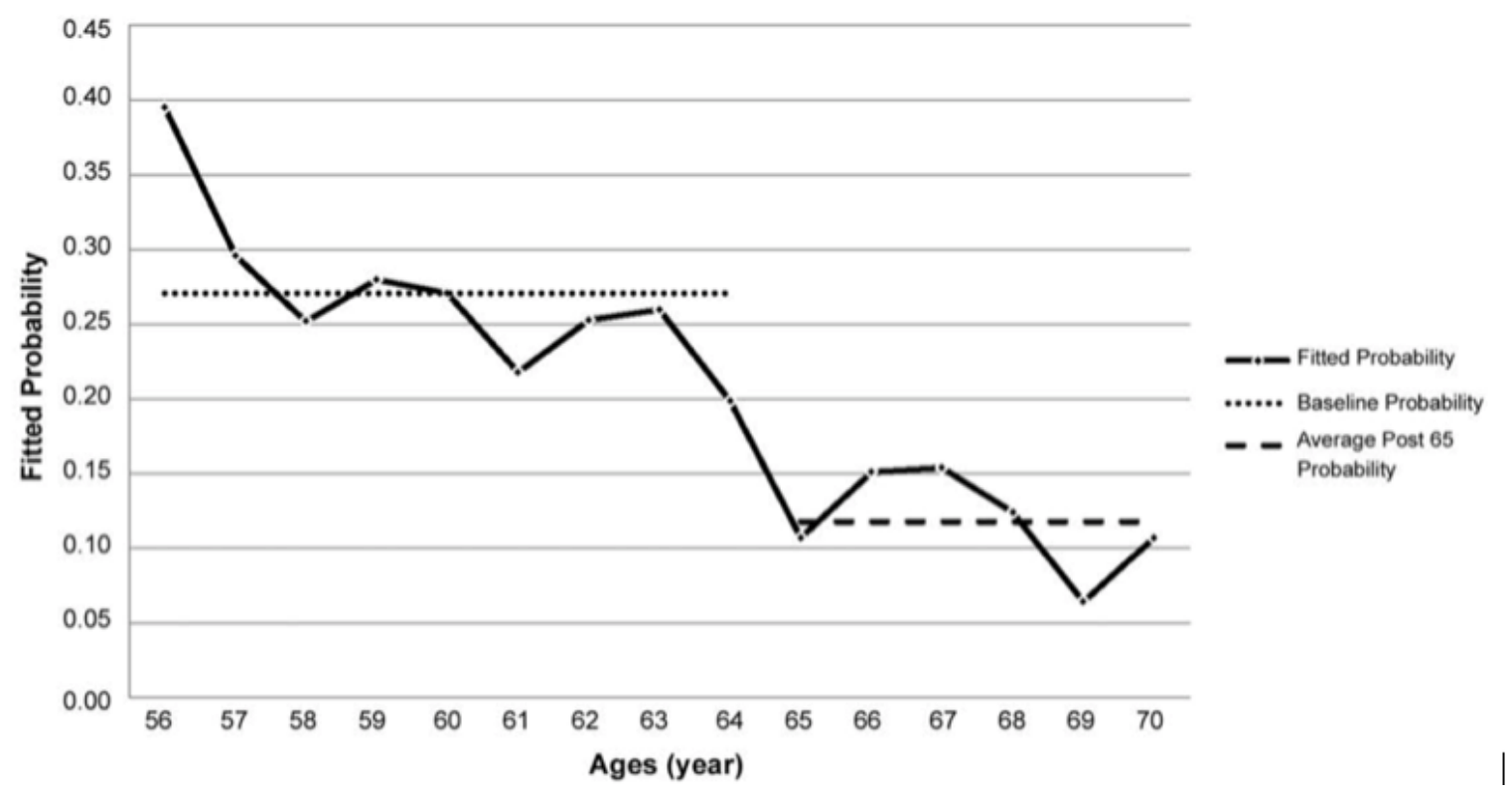

Figure 2: Fitted probability of food insecurity by age from probit regression (Canadian Community Health Survey pooled data 2007-2013 [9]).

economically disadvantaged are currently insufficient in preventing food insecurity. $70 \%$ of households dependent on social assistance report an inability to reliably put food on the table, highlighting the inadequacies of these programs to meet basic needs [1]. However, research has shown that improvements to social assistance can be a promising strategy to provide households with basic needs. For example, British Columbia's modest increase in social assistance rates in 2005 resulted in a significantly lower prevalence of food insecurity, highlighting sensitivity to small increases in income [8]. Meanwhile, $62 \%$ of food insecure households in Canada rely on income from employment, mostly in the form of low-wage, precarious jobs. This demonstrates the inability of the majority of food insecure individuals to garner a living wage, despite participation in the workforce.

As food insecurity is strongly rooted in inadequate income, a guaranteed annual income holds promise to reduce material deprivation. In fact, an example of a guaranteed income has already been implemented in Canada for seniors. When an adult turns 65 , they become eligible for the Old Age Security and Guaranteed Income Supplement programmes. After the age of 65 , the prevalence of food insecurity among low-income individuals drops by half, partly due to the significant protection offered by these guaranteed incomes (Figure 2) [9]. Expansion of a guaranteed income may similarly reduce food insecurity rates in Canada, with notable impacts on chronic disease prevalence and the ability for individuals to better manage their health.

Despite relative affluence in Canada, food insecurity continues to be a public health concern that has remained largely unaddressed. Lagging action on this social determinant of chronic disease will continue to burden the health of individuals and the Canadian healthcare system. Along with bold efforts to achieve scientific breakthroughs in disease treatment throughout Canada, the fundamentals of providing basic needs in every household should not remain forgotten as an important strategy to reduce the incidence and burden of chronic disease.

\section{References}

[1] Tarasuk, V., Mitchell, A., Dachner, N. (2014) Household food insecurity in Canada, 2012. Toronto, ON: Research to Identify Policy Options to Reduce Food Insecurity (PROOF).

[2] Kirkpatrick, S. I., Mclntyre, L., Potestio, M. L. (2010) Child hunger and long-term adverse consequences for health. Arch Pediatr Adolesc Med., 164 (8), 754-762.

[3] Tarasuk, V., Mitchell, A., McLaren, L., Mclntyre, L. (2013) Chronic physical and mental health conditions among adults may increase vulnerability to household food insecurity. J Nutr., 143 (11), 1785-1793.

[4] Tarasuk, V., Cheng, J., de Oliveira, C., Dachner, N., Gundersen, C., Kurdyak, P. (2015) Association between household food insecurity and annual health care costs. CMAJ, 187 (14), E429-E436.

[5] Fitzpatrick, T., Rosella, L. C., Calzavara, A., Petch, J., Pinto, A. D., Manson, H., et al. (2015) Looking Beyond Income and Education: Socioeconomic Status Gradients Among Future High-Cost Users of Health Care. Am J Prev Med., 49 (2), 161-171. 
[6] Loopstra, R., Tarasuk, V. (2015) Food bank usage is a poor indicator of food insecurity: Insights from Canada. Social Policy and Society, 14 (3), 443-455.

[7] Loopstra, R., Tarasuk, V. (2012) The Relationship between Food Banks and Household Food Insecurity among Low-Income Toronto Families. Canadian Public Policy, 38 (4), 497-514.
[8] Li, N., Dachner, N., Tarasuk, V. (2016) The impact of changes in social policies on household food insecurity in British Columbia, 2005-2012. Prev Med., 93, 151-158.

[9] Mclntyre, L., Dutton, D. J., Kwok, C., Emery, J. C. H. (2016) Reduction of Food Insecurity among Low-Income Canadian Seniors as a Likely Impact of a Guaranteed Annual Income. Canadian Public Policy, 42 (3), 274286.

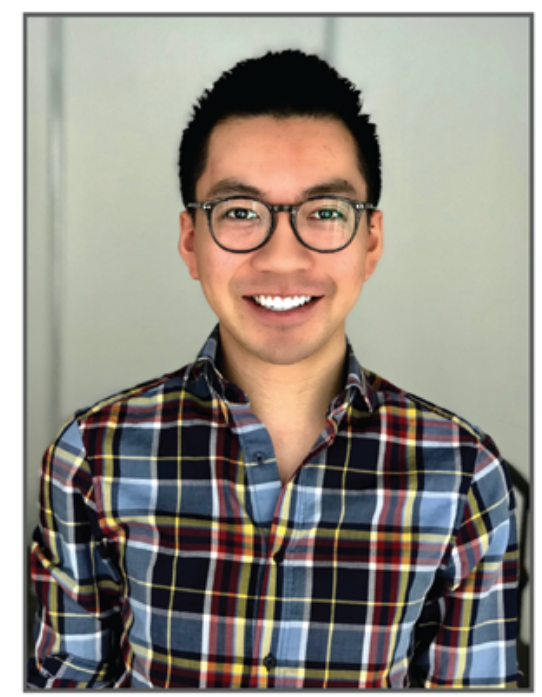

Jordan Mak completed his BSc in Nutritional Sciences at McGill University and has practiced as a Registered Dietitian at the University Health Network for 5 years. He is currently completing his MSc in Nutritional Sciences at the University of Toronto under the supervision of Prof. Valerie Tarasuk. His research focuses on household food insecurity in Canada amongst vulnerable populations. 